\title{
Some Hermite-Hadamard-Fejer type inequalities for harmonically convex functions via fractional integral
}

\author{
Imdat Iscan ${ }^{1}$, Sercan Turhan ${ }^{2}$ and Selahattin Maden ${ }^{3}$ \\ ${ }^{1}$ Department of Mathematics, Giresun University, Giresun, Turkey \\ ${ }^{2}$ Dereli Vocational High School, Giresun University, Giresun, Turkey \\ ${ }^{3}$ Department of Mathematics, Ordu University, Ordu, Turkey
}

Received: 13 November 2015, Revised: 19 November 2015, Accepted: 29 December 2015

Published online: 30 March 2016

\begin{abstract}
In this paper, we gave the new general identity for differentiable functions. As a result of this identity some new and general inequalities for differentiable harmonically-convex functions are obtained.
\end{abstract}

Keywords: Harmoically-convex, Hermite-Hadamard-Fejer type inequality, fractional integral.

\section{Introduction}

The classical or the usual convexity is defined as follows,

Definition 1. A function $f: I \longrightarrow \mathbb{R}, \emptyset \neq I \subseteq \mathbb{R}$, is said to be convex on I if inequality

$$
f(t x+(1-t) y) \leq t f(x)+(1-t) f(y)
$$

holds for all $x, y \in I$ and $t \in[0,1]$.

A number of papers have been written on inequalities using the classical convexty and one of the most captivating inequalities in mathematical analysis is stated as follows,

$$
f\left(\frac{a+b}{2}\right) \leq \frac{1}{b-a} \int_{a}^{b} f(x) d x \leq \frac{f(a)+f(b)}{2}
$$

where $f: I \subseteq \mathbb{R} \longrightarrow$ be a convex mapping and $a, b \in I$ with $a \leq b$. Both the inequalities hold in reversed direction if $f$ is concave. The inequalities stated in (1) are known as Hermite-Hadamard inequalities.

For more results on (1) which provide new proof, significantly extensions, generalizations, refinements, counterparts, new Hermite-Hadamard-type inequalities and numerous applications, we refer the interested reader to $[2,3,5,6,8,9,12$, $13,15,16]$ and the references there in.

The usual notion of convex function have been generalized in diverse manners. One of them is the so called harmonically s-convex functions and is stated in the definition below. 
Definition 2. [5,7] Let $I \subset(0, \infty)$ be a real interval. A function $f: I \longrightarrow \mathbb{R}$ is said to be harmonically s-convex(concave), if

$$
f\left(\frac{x y}{t x+(1-t) y}\right) \leq(\geq) t^{s} f(y)+(1-t)^{s} f(x)
$$

holds for all $x, y \in I$ and $t \in[0,1]$, and for some fixed $s \in(0,1]$.

It can be easily seen that for $s=1$ in Defination 2 reduces to following Defination 3,

Definition 3. [6] A function $f: I \subseteq \mathbb{R} \backslash\{0\} \longrightarrow \mathbb{R}$ is said to be harmonically-convex function, if

$$
f\left(\frac{x y}{t x+(1-t) y}\right) \leq t f(y)+(1-t) f(x)
$$

holds for all $x, y \in I$ and $t \in[0,1]$. If the inequality is reversed, then $f$ is said to be harmonically concave.

Proposition 1. [6] Let $I \subset \mathbb{R} \backslash\{0\}$ be a real interval and $f: I \rightarrow \mathbb{R}$ is function, then:

(i) if $I \subset(0, \infty)$ and $f$ is convex and nondecreasing function then $f$ is harmonically convex.

(ii) if $I \subset(0, \infty)$ and $f$ is harmonically convex and nonincreasing function then $f$ is convex.

(iii) if $I \subset(-\infty, 0)$ and $f$ is harmonically convex and nondecreasing function then $f$ is convex.

(iv) if $I \subset(-\infty, 0)$ and $f$ is convex and nonincreasing function then $f$ is harmonically convex.

For the properties of harmonically-convex functions and harmonically-s-convex function, we refer the reader to [1,5,6, 7 , $8,10,11]$ and the reference there in.

Most recently, a number of findings have been seen on Hermite-Hadamard type integral inequalities for harmonically-convex and for harmonically-s-convex functions.

In [14], Fejér established the following Fejér inequality which is the weighted generalization of Hermite-Hadamard inequality (1).

Theorem 1. Let $f:[a, b] \longrightarrow \mathbb{R}$ be convex function. Then the inequality

$$
f\left(\frac{a+b}{2}\right) \int_{a}^{b} g(x) d x \leq \int_{a}^{b} f(x) g(x) d x \leq \frac{f(a)+f(b)}{2} \int_{a}^{b} g(x) d x,
$$

holds, where $g:[a, b] \longrightarrow \mathbb{R}$ is nonnegative, integrable and symmetric to $(a+b) / 2$.

For some results which generalize, improve, and extend the inequalities (1) and (2) see [15].

In [6], İşcan gave defination of harmonically convex functions and established following Hermite- Hadamard type inequality for harmonically convex functions as follows.

Theorem 2. [15] Let $f: I \subset \mathbb{R} \backslash\{0\} \rightarrow \mathbb{R}$ be a harmonically convex function and $a, b \in I$ with $a<b$. If $f \in L[a, b]$ then the following inequalities hold:

$$
f\left(\frac{2 a b}{a+b}\right) \leq \frac{a b}{b-a} \int_{a}^{b} \frac{f(x)}{x^{2}} d x \leq \frac{f(a)+f(b)}{2} .
$$

In [11], Iscan and Wu represented Hermite-Hadamard's inequalities for harmonically convex functions in fractional integral form as follows. 
Theorem 3. [11] Let $f: I \subseteq \mathbb{R}^{+} \rightarrow \mathbb{R}$ be a function such that $f \in L[a, b]$, where $a, b \in I$ with $a<b$. If $f$ is harmonicallyconvex on $[a, b]$, then the following inequalities for fractional integrals hold:

$$
f\left(\frac{2 a b}{a+b}\right) \leq \frac{\Gamma(\alpha+1)}{2}\left(\frac{a b}{b-a}\right)^{\alpha}\left\{J_{1 / a^{-}}^{\alpha}(f \circ h)(1 / b)+J_{1 / b^{+}}^{\alpha}(f \circ h)(1 / a)\right\} \leq \frac{f(a)+f(b)}{2},
$$

with $\alpha>0$ and $h(x)=1 / x$.

Definition 4. A function $g:[a, b] \subseteq \mathbb{R} \backslash\{0\} \longrightarrow \mathbb{R}$ is said to be harmonically symmetric with respect to $2 a b / a+b$ if

$$
g(x)=g\left(\frac{1}{\frac{1}{a}+\frac{1}{b}-\frac{1}{x}}\right)
$$

holds for all $x \in[a, b]$.

Theorem 4. In [1] Chan and Wu represented Hermite-Hadamard-Fejer inequality for harmonically convex functions as follows:

Theorem 5. Suppose that $f: I \subseteq \mathbb{R} \backslash\{0\} \longrightarrow \mathbb{R}$ be harmonically-convex function and $a, b \in I$, with $a<b$. If $f \in L[a, b]$ and $g:[a, b] \subseteq \mathbb{R} \backslash\{0\} \longrightarrow \mathbb{R}$ is nonnegative, integrable and harmonically symmetric with respect to $2 a b / a+b$, then

$$
f\left(\frac{2 a b}{a+b}\right) \int_{a}^{b} \frac{g(x)}{x^{2}} d x \leq \int_{a}^{b} \frac{f(x) g(x)}{x^{2}} d x \leq \frac{f(a)+f(b)}{2} \int_{a}^{b} \frac{g(x)}{x^{2}} d x
$$

In [10] Isscan and Kunt represented Hermite-Hadamard-Fejer type inequality for harmonically convex functions in fractional integral forms and established following identity as follows:

Theorem 6. Let $f:[a, b] \longrightarrow \mathbb{R}$ be harmonically convex function with $a<b$ and $f \in L[a, b]$. If $g:[a, b] \longrightarrow \mathbb{R}$ is nonnegative, integrable and harmonically symmetric with respect to $2 a b / a+b$, then the following inequalities for fractional integrals hold:

$$
\begin{aligned}
f\left(\frac{2 a b}{a+b}\right)\left[J_{1 / a^{-}}^{\alpha}(g \circ h)(1 / b)+J_{1 / b^{+}}^{\alpha}(g \circ h)(1 / a)\right] & \leq\left[J_{1 / a^{-}}^{\alpha}(f g \circ h)(1 / b)+J_{1 / b^{+}}^{\alpha}(f g \circ h)(1 / a)\right] \\
& \leq \frac{f(a)+f(b)}{2}\left[J_{1 / a^{-}}^{\alpha}(g \circ h)(1 / b)+J_{1 / b^{+}}^{\alpha}(g \circ h)(1 / a)\right]
\end{aligned}
$$

with $\alpha>0$ and $h(x)=1 / x, x \in\left[\frac{1}{b}, \frac{1}{a}\right]$.

Definition 5. Let $f \in L[a, b]$. The right-hand side and left-hand side Hadamard fractional integrals $J_{a^{+}}^{\alpha} f$ and $J_{b^{-}}^{\alpha} f$ of order $\alpha>0$ with $a \geq 0$ are defined by

$$
\begin{aligned}
& J_{a^{+}}^{\alpha} f(x)=\frac{1}{\Gamma(\alpha)} \int_{a}^{x}(x-t)^{\alpha-1} f(t) d t, x>a \\
& J_{b^{-}}^{\alpha} f(x)=\frac{1}{\Gamma(\alpha)} \int_{x}^{b}(t-x)^{\alpha-1} f(t) d t, x<b
\end{aligned}
$$

respectively where $\Gamma(\alpha)$ is the Gamma function defined by $\Gamma(\alpha)=\int_{0}^{\infty} e^{-t} t^{\alpha-1}$ and $J_{a^{+}}^{0} f(x)=J_{b^{-}}^{0} f(x)=f(x)$

Lemma 1. For $0<\theta \leq 1$ and $0<a \leq b$ we have

$$
\left|a^{\theta}-b^{\theta}\right| \leq(b-a)^{\theta}
$$


In [4] D. Y. Hwang found out a new identity and by using this identity, established a new inequalities. Then in [12] İ. Isscan and S. Turhan used this identity for GA-convex functions and obtain generalized new inequalities. In this paper, we established a new inequality similar to inequality in [12] and then we obtained some new and general integral inequalities for differentiable harmonically-convex functions using this lemma. The following sections, let the notion, $L(t)=\frac{a H}{t H+(1-t) a}, U(t)=\frac{b H}{t H+(1-t) b}$ and $H=H(a, b)=\frac{2 a b}{a+b}$.

\section{Main result}

Throughout this section, let $\|g\|_{\infty}=\sup _{t \in[a, b]}|g(x)|$, for the continuous function $g:[a, b] \longrightarrow[0, \infty)$ be differentiable mapping $I^{o}$, where $a, b \in I$ with $a \leq b$, and $h:[a, b] \longrightarrow[0, \infty)$ be differentiable mapping.

Lemma 2. If $f^{\prime} \in L[a, b]$ then the following inequality holds:

$$
\begin{aligned}
& {[h(b)-2 h(a)] \frac{f(a)}{2}+h(b) \frac{f(b)}{2}-\int_{a}^{b} f(x) h^{\prime}(x) d x} \\
& =\frac{b-a}{4 a b}\left\{\int_{0}^{1}[2 h(L(t))-h(b)] f^{\prime}(L(t))(L(t))^{2} d t+\int_{0}^{1}[2 h(U(t))-h(b)] f^{\prime}(U(t))(U(t))^{2} d t\right\} .
\end{aligned}
$$

Proof. By the integration by parts, we have

$$
I_{1}=\int_{0}^{1}[2 h(L(t))-h(b)] d(f(L(t)))=\left.[2 h(L(t))-h(b)] f(L(t))\right|_{0} ^{1}-\left(\frac{1}{a}-\frac{1}{b}\right) \int_{0}^{1} f(L(t)) h^{\prime}(L(t))(L(t))^{2} d t
$$

and

$$
I_{2}=\int_{0}^{1}[2 h(U(t))-h(b)] d(f(U(t)))=\left.[2 h(U(t))-h(b)] f(U(t))\right|_{0} ^{1}-\left(\frac{1}{a}-\frac{1}{b}\right) \int_{0}^{1} f(U(t)) h^{\prime}(U(t))(U(t))^{2} d t
$$

Therefore

$$
\frac{I_{1}+I_{2}}{2}=[h(b)-2 h(a)] \frac{f(a)}{2}+h(b) \frac{f(b)}{2}-\frac{b-a}{2 a b}\left\{\int_{0}^{1} f(L(t)) h^{\prime}(L(t))(L(t))^{2} d t+\int_{0}^{1} f(U(t)) h^{\prime}(U(t))(U(t))^{2} d t\right\} .
$$

This complete the proof.

Lemma 3. For $a, H, b>0$, we have

$$
\begin{aligned}
& \zeta_{1}(a, b)=\int_{0}^{1}|2 h(L(t))-h(b)|(1-t)(L(t))^{2} d t \\
& \zeta_{2}(a, b)=\int_{0}^{1} t(L(t))^{2}|2 h(L(t))-h(b)| d t+\int_{0}^{1} t\left((U(t))^{2}|2 h(U(t))-h(b)| d t\right. \\
& \zeta_{3}(a, b)=\int_{0}^{1}|2 h(U(t))-h(b)|(1-t)(U(t))^{2} d t .
\end{aligned}
$$


Theorem 7. Let $f: I \subseteq \mathbb{R}=(0, \infty) \longrightarrow \mathbb{R}$ be differentiable mapping $I^{o}$, where $a, b \in I$ with $a<b$. If the mapping $\left|f^{\prime}\right|$ is harmonically-convex on $[a, b]$, then the following inequality holds:

$$
\left|[h(b)-2 h(a)] \frac{f(a)}{2}+h(b) \frac{f(b)}{2}-\int_{a}^{b} f(x) h^{\prime}(x) d x\right| \leq \frac{b-a}{4 a b}\left[\zeta_{1}(a, b)\left|f^{\prime}(a)\right|+\zeta_{2}(a, b)\left|f^{\prime}(H)\right|+\zeta_{3}(a, b)\left|f^{\prime}(b)\right|\right]
$$

where $\zeta_{1}(a, b), \zeta_{2}(a, b), \zeta_{3}(a, b)$ are defined in Lemma 3.

Proof. Continuing equality (8) in Lemma 2

$$
\begin{aligned}
& \left|[h(b)-2 h(a)] \frac{f(a)}{2}+h(b) \frac{f(b)}{2}-\int_{a}^{b} f(x) h^{\prime}(x) d x\right| \\
& \leq \frac{b-a}{4 a b}\left\{\int_{0}^{1}|2 h(L(t))-h(b)|\left|f^{\prime}(L(t))(L(t))^{2}\right| d t+\int_{0}^{1}|2 h(U(t))-h(b)|\left|f^{\prime}(U(t))(U(t))^{2}\right| d t\right\} .
\end{aligned}
$$

Using $\left|f^{\prime}\right|$ is harmoncally-convex in (14).

$$
\begin{aligned}
& \left|[h(b)-2 h(a)] \frac{f(a)}{2}+h(b) \frac{f(b)}{2}-\int_{a}^{b} f(x) h^{\prime}(x) d x\right| \leq \frac{b-a}{4 a b}\left\{\int_{0}^{1}|2 h(L(t))-h(b)|\left\{t\left|f^{\prime}(H)\right|+(1-t)\left|f^{\prime}(a)\right|\right\}(L(t))^{2} d t\right. \\
& \left.+\int_{0}^{1}|2 h(U(t))-h(b)|\left\{t\left|f^{\prime}(H)\right|+(1-t)\left|f^{\prime}(b)\right|\right\}(U(t))^{2} d t\right\}
\end{aligned}
$$

by (15) and Lemma 2, this proof is complete.

Corollary 1. Let $h(t)=\int_{1 / t}^{1 / a}\left[\left(x-\frac{1}{b}\right)^{\alpha-1}+\left(\frac{1}{a}-x\right)^{\alpha-1}\right] g \circ \varphi(x) d x$ for all $1 / t \in\left[\frac{1}{b}, \frac{1}{a}\right], \alpha>0$ and $g:[a, b] \longrightarrow[0, \infty)$ be continuous positive mapping and symmetric to $\frac{2 a b}{a+b}$ in Teorem 7 , we obtain:

$$
\begin{gathered}
\left|\left(\frac{f(a)+f(b)}{2}\right)\left[J_{1 / b^{+}}^{\alpha} g \circ \varphi(1 / a)+J_{1 / a^{-}}^{\alpha} g \circ \varphi(1 / b)\right]-\left[J_{1 / b^{+}}^{\alpha}(f g \circ \varphi)(1 / a)+J_{1 / a^{-}}^{\alpha}(f g \circ \varphi)(1 / b)\right]\right| \\
\leq \frac{(b-a)^{\alpha+1}\|g\|_{\infty}}{2^{\alpha+1}(a b)^{\alpha+1} \Gamma(\alpha+1)}\left[C_{1}(\alpha)\left|f^{\prime}(a)\right|+C_{2}(\alpha)\left|f^{\prime}(H)\right|+C_{3}(\alpha)\left|f^{\prime}(b)\right|\right]
\end{gathered}
$$

where

$$
\begin{aligned}
& C_{1}(\alpha)=\int_{0}^{1}(1-t)\left[(1+t)^{\alpha}-(1-t)^{\alpha}\right](L(t))^{2} d t \\
& C_{2}(\alpha)=\int_{0}^{1} t\left[(1+t)^{\alpha}-(1-t)^{\alpha}\right]\left[(L(t))^{2}+(U(t))^{2}\right] d t \\
& C_{3}(\alpha)=\int_{0}^{1}(1-t)\left[(1+t)^{\alpha}-(1-t)^{\alpha}\right](L(t))^{2} d t .
\end{aligned}
$$


Specially in (16) and using Lemma 1, for $0<\alpha \leq 1$ we have:

$$
\begin{gathered}
\left|\left(\frac{f(a)+f(b)}{2}\right)\left[J_{1 / b^{+}}^{\alpha} g \circ \varphi(1 / a)+J_{1 / a^{-}}^{\alpha} g \circ \varphi(1 / b)\right]-\left[J_{1 / b^{+}}^{\alpha}(f g \circ \varphi)(1 / a)+J_{1 / a^{-}}^{\alpha}(f g \circ \varphi)(1 / b)\right]\right| \\
\leq \frac{(b-a)^{\alpha+1}\|g\|_{\infty}}{2(a b)^{\alpha+1} \Gamma(\alpha+1)}\left[C_{1}(\alpha)\left|f^{\prime}(a)\right|+C_{2}(\alpha)\left|f^{\prime}(H)\right|+C_{3}(\alpha)\left|f^{\prime}(b)\right|\right]
\end{gathered}
$$

where

$$
C_{1}(\alpha)=\int_{0}^{1}(1-t) t^{\alpha}(L(t))^{2} d t, C_{2}(\alpha)=\int_{0}^{1} t^{\alpha+1}\left[(L(t))^{2}+(U(t))^{2}\right] d t, C_{3}(\alpha)=\int_{0}^{1}(1-t) t^{\alpha}(U(t))^{2} d t
$$

Proof. By left side of inequality (15) in Teorem 7, when we write $h(t)=\int_{1 / t}^{1 / a}\left[\left(x-\frac{1}{b}\right)^{\alpha-1}+\left(\frac{1}{a}-x\right)^{\alpha-1}\right] g \circ \varphi(x) d x$ for all $x \in[1 / b, 1 / a]$ and $\varphi(x)=1 / x$, we have

$$
\left|\Gamma(\alpha)\left(\frac{f(a)+f(b)}{2}\right)\left[J_{1 / b^{+}}^{\alpha} g \circ \varphi(1 / a)+J_{1 / a^{-}}^{\alpha} g \circ \varphi(1 / b)\right]-\Gamma(\alpha)\left[J_{1 / b^{+}}^{\alpha}(f g \circ \varphi)(1 / a)+J_{1 / a^{-}}^{\alpha}(f g \circ \varphi)(1 / b)\right]\right| .
$$

On the other hand, right side of inequality (15), with

$$
\begin{aligned}
\Psi(x, a, b) & =\left(x-\frac{1}{b}\right)^{\alpha-1}+\left(\frac{1}{a}-x\right)^{\alpha-1} \\
& \leq \frac{b-a}{4 a b}\left\{\int_{0}^{1}\left|2 \int_{1 / L(t)}^{1 / a}[\Psi(x, a, b)] g \circ \varphi(x) d x-\int_{1 / b}^{1 / a}[\Psi(x, a, b)] g \circ \varphi(x) d x\right|\left\{t\left|f^{\prime}(H)\right|+(1-t)\left|f^{\prime}(a)\right|\right\}(L(t))^{2} d t\right. \\
& \left.+\int_{0}^{1}\left|2 \int_{1 / U(t)}^{1 / a}[\Psi(x, a, b)] g \circ \varphi(x) d x-\int_{1 / b}^{1 / a}[\Psi(x, a, b)] g \circ \varphi(x) d x\right|\left\{t\left|f^{\prime}(H)\right|+(1-t)\left|f^{\prime}(b)\right|\right\}(U(t))^{2} d t\right\} .
\end{aligned}
$$

Since $g(x)$ is symmetric to $x=\frac{2 a b}{a+b}$, we have

$$
\left|2 \int_{1 / L(t)}^{1 / a}[\Psi(x, a, b)] g \circ \varphi(x) d x-\int_{1 / b}^{1 / a}[\Psi(x, a, b)](g \circ \varphi)(x) d x\right|=\left|\int_{1 / U(t)}^{1 / L(t)}[\Psi(x, a, b)](g \circ \varphi)(x) d x\right|
$$

and

$$
\left|2 \int_{1 / U(t)}^{1 / a}[\Psi(x, a, b)] g \circ \varphi(x) d x-\int_{1 / b}^{1 / a}[\Psi(x, a, b)](g \circ \varphi)(x) d x\right|=\left|\int_{1 / U(t)}^{1 / L(t)}[\Psi(x, a, b)](g \circ \varphi)(x) d x\right|
$$

for all $t \in[0,1]$. By (18)- (20), we have

$$
\begin{gathered}
\left|\left(\frac{f(a)+f(b)}{2}\right)\left[J_{1 / b^{+}}^{\alpha} g \circ \varphi(1 / a)+J_{1 / a^{-}}^{\alpha} g \circ \varphi(1 / b)\right]-\left[J_{1 / b^{+}}^{\alpha}(f g \circ \varphi)(1 / a)+J_{1 / a^{-}}^{\alpha}(f g \circ \varphi)(1 / b)\right]\right| \\
\quad \leq \frac{b-a}{4 a b \Gamma(\alpha)}\left\{\int_{0}^{1}\left|\left[\int_{1 / U(t)}^{1 / L(t)} \Psi(x, a, b)\right] g \circ \varphi(x) d x\right|\left\{t\left|f^{\prime}(H)\right|+(1-t)\left|f^{\prime}(a)\right|\right\}(L(t))^{2} d t\right.
\end{gathered}
$$




$$
\begin{aligned}
+ & \left.\int_{0}^{1} \int_{1 / U(t)}^{1 / L(t)}[\Psi(x, a, b)] g \circ \varphi(x) d x \mid\left\{t\left|f^{\prime}(H)\right|+(1-t)\left|f^{\prime}(b)\right|\right\}(U(t))^{2} d t\right\} \\
\leq \frac{(b-a)\|g\|_{\infty}}{4 a b \Gamma(\alpha)} & \left\{\int_{0}^{1}\left[\int_{1 / U(t)}^{1 / L(t)}|\Psi(x, a, b)| d x\right]\left\{t\left|f^{\prime}(H)\right|+(1-t)\left|f^{\prime}(a)\right|\right\}(L(t))^{2} d t\right. \\
+ & \left.\int_{0}^{1}\left[\int_{1 / U(t)}^{1 / L(t)}|\Psi(x, a, b)| d x\right]\left\{t\left|f^{\prime}(H)\right|+(1-t)\left|f^{\prime}(b)\right|\right\}(U(t))^{2} d t\right\} .
\end{aligned}
$$

In the last inequality,

$$
\int_{1 / U(t)}^{1 / L(t)}|\Psi(x, a, b)| d x=\int_{1 / U(t)}^{1 / L(t)}\left(x-\frac{1}{b}\right)^{\alpha-1} d x+\int_{1 / U(t)}^{1 / L(t)}\left(\frac{1}{a}-x\right)^{\alpha-1} d x=\frac{2^{1-\alpha}}{\alpha}\left(\frac{b-a}{a b}\right)^{\alpha}\left\{(1+t)^{\alpha}-(1-t)^{\alpha}\right\} .
$$

By Lemma 1, we have

$$
\int_{1 / U(t)}^{1 / L(t)}|\Psi(x, a, b)| d x=\int_{1 / U(t)}^{1 / L(t)}\left(x-\frac{1}{b}\right)^{\alpha-1} d x+\int_{1 / U(t)}^{1 / L(t)}\left(\frac{1}{a}-x\right)^{\alpha-1} d x \leq \frac{2}{\alpha}\left(\frac{b-a}{a b}\right)^{\alpha} t^{\alpha}
$$

A combination of (21) and (22), we have (16). This complete is proof.

\section{Corollary 2. In Corollary 1,}

(i) If $\alpha=1$ is in corollary, we obtain following Hermite-Hadamard-Fejer Type inequality for harmonically-convex function which is related the left-hand side of (17):

$$
\left|\left[\frac{f(a)+f(b)}{2}\right] \int_{a}^{b} \frac{g(x)}{x^{2}} d x-\int_{a}^{b} f(x) \frac{g(x)}{x^{2}} d x\right| \leq \frac{(b-a)^{2}}{4(a b)^{2}}\|g\|_{\infty}\left[C_{1}(1)\left|f^{\prime}(a)\right|+C_{2}(1)\left|f^{\prime}(H)\right|+C_{3}(1)\left|f^{\prime}(b)\right|\right]
$$

where for $a, b, H>0$, we have

$$
\begin{aligned}
& C_{1}(1)=\int_{0}^{1}(1-t) t(L(t))^{2} d t \\
& C_{2}(1)=\int_{0}^{1} t^{2}\left[(L(t))^{2}+(U(t))^{2}\right] d t \\
& C_{3}(1)=\int_{0}^{1}(1-t) t(U(t))^{2} d t
\end{aligned}
$$


(ii) If $g(x)=1$ is in corollary, we obtain following Hermite-Hadamard-Fejer Type inequality for harmonically-convex function which is related the left-hand side of (16):

$$
\begin{aligned}
& \left|\left(\frac{f(a)+f(b)}{2}\right)-\frac{(a b)^{\alpha} \Gamma(\alpha+1)}{2(b-a)^{\alpha}}\left[J_{1 / b^{+}}^{\alpha}(f \circ \varphi)(1 / a)+J_{1 / a^{-}}^{\alpha}(f \circ \varphi)(1 / b)\right]\right| \\
& \leq \frac{(b-a)}{2^{\alpha+2} a b}\left[C_{1}(\alpha)\left|f^{\prime}(a)\right|+C_{2}(\alpha)\left|f^{\prime}(H)\right|+C_{3}(\alpha)\left|f^{\prime}(b)\right|\right] .
\end{aligned}
$$

(iii) If $g(x)=1$ and $\alpha=1$ is in corollary, we obtain following Hermite-Hadamard-Fejer Type inequality for harmonically-convex function which is related the left-hand side of (17):

$$
\left|\left(\frac{f(a)+f(b)}{2}\right)-\frac{a b}{(b-a)} \int_{a}^{b} \frac{f(x)}{x^{2}} d x\right| \leq \frac{(b-a)}{4(a b)}\left[C_{1}(1)\left|f^{\prime}(a)\right|+C_{2}(1)\left|f^{\prime}(H)\right|+C_{3}(1)\left|f^{\prime}(b)\right|\right] .
$$

Theorem 8. Let $f: I \subseteq \mathbb{R} \backslash\{0\} \longrightarrow \mathbb{R}$ be differentiable mapping $I^{o}$, where $a, b \in I$ with $a<b$. If the mapping $\left|f^{\prime}\right|^{q}$ is harmonically-convex on $[a, b]$, then the following inequality holds:

$$
\left|[h(b)-2 h(a)] \frac{f(a)}{2}+h(b) \frac{f(b)}{2}-\int_{a}^{b} f(x) h^{\prime}(x) d x\right| \leq \frac{b-a}{4 a b}\left\{\eta_{1}^{1-\frac{1}{q}} \times \eta_{2}^{\frac{1}{q}}+\eta_{3}^{1-\frac{1}{q}} \times \eta_{4}^{\frac{1}{q}}\right\}
$$

where

$$
\begin{aligned}
& \eta_{1}=\left(\int_{0}^{1}|2 h(L(t))-h(b)| d t\right) \\
& \eta_{2}=\left(\int_{0}^{1}(|2 h(L(t))-h(b)| d t) \times\left(t(L(t))^{2 q}\left|f^{\prime}(a)\right|^{q}+(1-t)(L(t))^{2 q}\left|f^{\prime}(H)\right|^{q}\right)\right) \\
& \eta_{3}=\left(\int_{0}^{1}|2 h(U(t))-h(b)| d t\right) \\
& \eta_{4}=\left(\int_{0}^{1}(|2 h(U(t))-h(b)| d t) \times\left(t(U(t))^{2 q}\left|f^{\prime}(a)\right|^{q}+(1-t)(U(t))^{2 q}\left|f^{\prime}(H)\right|^{q}\right)\right)
\end{aligned}
$$

Proof. Continuing from (14) in Theorem 7, we use Hölder Inequality and we use that $\left|f^{\prime}\right|^{q}$ is harmonically-convex. Thus this proof is complete.

Corollary 3. Let $h(t)=\int_{1 / t}^{1 / a}\left[\left(x-\frac{1}{b}\right)^{\alpha-1}+\left(\frac{1}{a}-x\right)^{\alpha-1}\right](g \circ \varphi)(x) d x$ for all $t \in[a, b]$ and $g:[a, b] \longrightarrow[0, \infty)$ be continuous positive mapping and symmetric to $\frac{2 a b}{a+b}$ in Teorem 8 , we obtain:

$$
\begin{aligned}
& \left|\left(\frac{f(a)+f(b)}{2}\right)\left[J_{1 / b^{+}}^{\alpha}(g \circ \varphi)(1 / a)+J_{1 / a^{-}}^{\alpha}(g \circ \varphi)(1 / b)\right]-\left[J_{1 / b^{+}}^{\alpha}(f g \circ \varphi)(1 / a)+J_{1 / a^{-}}^{\alpha}(f g \circ \varphi)(1 / b)\right]\right| \\
& \leq \frac{(b-a)^{\alpha+1}\|g\|_{\infty}}{2^{\alpha+1}(a b)^{\alpha+1} \Gamma(\alpha+1)}\left(\frac{2^{2}\left(2^{\alpha}-1\right)}{\alpha+1}\right)^{1-\frac{1}{q}}\left[C_{1}(\alpha, q)\left|f^{\prime}(a)\right|^{q}+C_{2}(\alpha, q)\left|f^{\prime}(H)\right|^{q}+C_{3}(\alpha, q)\left|f^{\prime}(b)\right|^{q}\right]^{\frac{1}{q}}
\end{aligned}
$$


where for $q>1$

$$
\begin{aligned}
& C_{1}(\alpha, q)=\int_{0}^{1}\left[(1+t)^{\alpha}-(1-t)^{\alpha}\right] t(L(t))^{2 q} d t \\
& C_{2}(\alpha, q)=\int_{0}^{1}\left[(1+t)^{\alpha}-(1-t)^{\alpha}\right](1-t)\left((L(t))^{2 q}+(U(t))^{2 q}\right) d t \\
& C_{3}(\alpha, q)=\int_{0}^{1}\left[(1+t)^{\alpha}-(1-t)^{\alpha}\right] t(U(t))^{2 q} d t .
\end{aligned}
$$

Proof. Continuing from (22) of Corollary 1 and (26) in Theorem 8,

$$
\begin{aligned}
\left|\left(\frac{f(a)+f(b)}{2}\right)\left[\zeta_{1}\right]-\left[\zeta_{2}\right]\right| & \leq \frac{(b-a)^{\alpha+1}}{2^{\alpha+1} \Gamma(\alpha+1)}\left\{\ell_{1} \times \ell_{2}+\ell_{1} \times \ell_{3}\right\} \\
& \leq \frac{(b-a)^{\alpha+1}\|g\|_{\infty}}{2^{\alpha+1}(a b)^{\alpha+1} \Gamma(\alpha+1)}\left(\zeta_{0}\right)^{1-\frac{1}{q}}\left[\ell_{2}+\ell_{3}\right]
\end{aligned}
$$

where

$$
\begin{aligned}
& \zeta_{0}=\frac{2^{\alpha+1}-2}{\alpha+1}, \\
& \zeta_{1}=J_{a^{+}}^{\alpha} g(b)+J_{b^{-}}^{\alpha} g(a), \\
& \zeta_{2}=J_{a^{+}}^{\alpha}(f g)(b)+J_{b^{-}}^{\alpha}(f g)(a), \\
& \ell_{1}=\left(\int_{0}^{1}\left[(1+t)^{\alpha}-(1-t)^{\alpha}\right] d t\right)^{1-\frac{1}{q}}, \\
& \ell_{2}=\left(\int_{0}^{1}\left[(1+t)^{\alpha}-(1-t)^{\alpha}\right]\left(t(L(t))^{2 q}\left|f^{\prime}(a)\right|^{q}+(1-t)(L(t))^{2 q}\left|f^{\prime}(H)\right|^{q}\right) d t\right)^{\frac{1}{q}}, \\
& \ell_{3}=\left(\int_{0}^{1}\left[(1+t)^{\alpha}-(1-t)^{\alpha}\right]\left(t(U(t))^{2 q}\left|f^{\prime}(b)\right|^{q}+(1-t)(U(t))^{2 q}\left|f^{\prime}(H)\right|^{q}\right) d t\right)^{\frac{1}{q}},
\end{aligned}
$$

By the power-mean inequality $\left(a^{r}+b^{r}<2^{1-r}(a+b)^{r}\right.$ for $\left.\quad a>0, b>0, \quad r<1\right)$ and $\frac{1}{p}+\frac{1}{q}=1$ we have

$$
\frac{(b-a)^{\alpha+1}\|g\|_{\infty}}{2^{\alpha+1}(a b)^{\alpha+1} \Gamma(\alpha+1)}\left(\zeta_{0}\right)^{1-\frac{1}{q}}\left[\ell_{4}+\ell_{5}\right] \leq \frac{(b-a)^{\alpha+1}\|g\|_{\infty}}{2^{\alpha+1}(a b)^{\alpha+1} \Gamma(\alpha+1)}\left(\frac{2^{2}\left(2^{\alpha}-1\right)}{\alpha+1}\right)^{\frac{1}{p}}\left[\int_{0}^{1}\left(\xi_{1}+\xi_{2}+\xi_{3}\right) d t\right]^{\frac{1}{q}},
$$

where

$$
\begin{aligned}
& \xi_{1}=\left[(1+t)^{\alpha}-(1-t)^{\alpha}\right] t(L(t))^{2 q}\left|f^{\prime}(a)\right|^{q}, \\
& \xi_{2}=\left[(1+t)^{\alpha}-(1-t)^{\alpha}\right](1-t)\left((L(t))^{2 q}+(U(t))^{2 q}\right)\left|f^{\prime}(H)\right|^{q}, \\
& \xi_{3}=\left[(1+t)^{\alpha}-(1-t)^{\alpha}\right] t(U(t))^{2 q}\left|f^{\prime}(b)\right|^{q} .
\end{aligned}
$$


Corollary 4. When $\alpha=1$ and $g(x)=1$ is taken in Corollary 3, we obtain:

$$
\left|\left(\frac{f(a)+f(b)}{2}\right)-\frac{a b}{(b-a)} \int_{a}^{b} \frac{f(x)}{x^{2}} d x\right| \leq \frac{(b-a)}{2^{2+\frac{1}{q}}(a b)}\left[C_{1}(1, q)\left|f^{\prime}(a)\right|^{q}+C_{2}(1, q)\left|f^{\prime}(H)\right|^{q}+C_{3}(1, q)\left|f^{\prime}(b)\right|^{q}\right]^{\frac{1}{q}} .
$$

This proof is complete.

\section{References}

[1] F. Chen and S. Wu, Hermite-Hadamard type inequalities for harmonically s-convex functions, Sci. World (2014), 7, Article ID 279158.

[2] Z. Dahmani, On Minkowski and Hermite-Hadamard integral inequalities via fractional integration, Ann. Funct. Anal. 1(1) (2010), pp. 51-58.

[3] S. S. Dragomir, Hermite Hadamard' s type inequalities for convex functions of selfadjoint operators in Hilbert spaces, Linear Algebra Appl. 436 (2012), no.5, 1503-1515.

[4] D-Y. Hwang, Some inequalities for differentiable convex mapping with application to weighted trapezoidal formula and higher moments of random variables, Applied Mathematics and Computation, 217 (2011), 9598-9605.

[5] I. Iscan, M. Kunt, Fejér and Hermite-Hadamard-Fejér type inequalities for harmonically $s$-convex functions via Fractional Integrals, The Australian Journal of Mathematical Analysis and Applications, (2015), Vol: 12, 1 ,Article 10, pp 1-6.

[6] I. Iscan, Hermite-Hadamard type inequalities for harmonically convex functions, Hacettepe Journal of Mathematics and Statistics 43 (6) (2014), 935-942.

[7] I. Iscan, Ostrowski type inequalities for harmonically s-convex functions, Konuralp Jurnal of Mathematics, Volume 3, No 1 (2015), pp. 63-74..

[8] I. Iscan, Some new general integral inequalities for h-convex and h-concave functions, Adv. Pure Appl. Math. 5(1) (2014), pp. 21-29 . doi: 10.1515/apam-2013-0029.

[9] I. Iscan, Generalization of different type integral inequalitiesfor s-convex functions via fractional integrals, Applicable Analysis, 2013. doi: 10.1080/00036811.2013.851785.

[10] I. Iscan, M. Kunt, Hermite-Hadamard-Fejer type inequalities for harmonically convex functions via fractional integrals, RGMIA Research Report Collection, 18(2015), Article 107, pp. 1-16.

[11] I. Iscan, S. Wu Hermite-Hadamard type inequalities for harmonically-convex functions via fractional integrals, Applied Mathematics and Computation, 238 (2014), 237-244.

[12] İ. İşcan, S. Turhan, Generalized Hermite-Hadamard-Fejer type inequalities for GA-convex functions via Fractional integral, arXiv:1511.03308v1 [math.CA], 10 Nov 2015.

[13] M.A. Latif, New Hermite Hadamard type integral inequalities for GA-convex functions with applications. Volume 34, Issue 4 (Nov 2014).

[14] L. Fejér, Uberdie Fourierreihen, II, Math. Naturwise. Anz. Ungar. Akad. , Wiss, 24 (1906), pp. 369-390, (in Hungarian)

[15] M. Z. Sarıkaya, On new Hermite Hadamard Fejér type integral inequalities, Stud. Univ. Babeş-Bolyai Math., 57(3) (2012), pp. 377-386.

[16] M. Z. Sarıkaya, E. Set, H. Yaldız and N. Başak, Hermite-Hadamard's inequalities for fractional integrals and related fractional inequalities, Mathematical and Computer Modelling, 57(9) (2013), pp. 2403-2407. 